\title{
Preparation and characterization of high-surface-area polymer substrates for microcalorimetry
}

\author{
V. A. Lee, ${ }^{1,2, *}$ R. G. Craig, ${ }^{1}$ F. E. Filisko, ${ }^{2}$ and R. Zand ${ }^{3}$ \\ ${ }^{1}$ Biologic and Materials Sciences, Dental School, ${ }^{2}$ Materials Science and Engineering Department, and ${ }^{3}$ Biophysics \\ Research Division, University of Michigan, Ann Arbor, Michigan 48109
}

The preparation and characterization of high-surface-area polymeric substrates suitable for the microcalorimetry of protein adsorption are described. High-surface-area polystyrene, poly(styrene-co-butyl methacrylate) and poly(styreneco-allyl alcohol) were prepared by adsorbing polymer from solution onto fumed silica. Verification of adsorption of polystyrene by silica was determined by noting peak shifts of the surface silanol group in the infrared. The amount of polymer adsorbed was determined from adsorption isotherms. The minimum thickness of polystyrene required to mask silicon oxide properties was found to be that thickness at which contact angles became constant, about 35 A. Polymer densities were measured. Water contact angles on each polymer surface indicate that poly(styrene-co-allyl alcohol) has the surface most wettable by water. Polymer-water interfacial energies were estimated from pendant drop results and a harmonic mean equation along with contact angles. Two methods were used to estimate the polar and dispersion components of the three polymers. Both methods predicted polystyrene to have the highest interfacial energy against water, and one method predicted poly(styrene-co-allyl alcohol) to have the lowest. A Wilhelmy plate study verified the change in interfacial properties as a function of contact time with water. A study of the heats of adsorption of lysozyme by each substrate using a modified Tien-Calvet microcalorimeter demonstrated the suitability of the substrates for microcalorimetry. (C) 1996 John Wiley \& Sons, Inc.

\section{INTRODUCTION}

The majority of materials currently in use as bloodcontacting implants are polymeric. The interaction of proteins and other components with the polymer surface generates various heats which can be measured in a microcalorimeter. Study of the pattern of heats generated may yield information about changes in protein conformation upon adsorption. Since the enthalpies involved in adsorption are extremely small, substrates of high surface area, generally $>70 \mathrm{~m}^{2} / \mathrm{g}$, allow the interaction of a large number of protein molecules with the surface and an increase in the accuracy of measurement.

The use of high-surface-area polymer substrates in the form of microspheres was first reported in the 1960s as supports for peptide synthesis. ${ }^{1}$ The use of polymer microspheres was expanded throughout the 1970s to include applications in cell separation, immunoassay, hemoperfusion, and extracorporeal therapy. ${ }^{2}$ Exten-

* To whom correspondence should be addressed at Biologic and Materials Sciences, Dental School, University of Michigan, Ann Arbor, MI 48109. sive research to design microspheres suitable for targeted drug delivery is currently under way.,4

Spherical polymer particles in the size range $50 \mu \mathrm{m}$ to $2 \mathrm{~mm}$ are usually produced by suspension, emulsion, or dispersion polymerization. Particles with functional groups at the surface are generally produced by copolymerization of functionalized monomers or by functionalization of preformed particles. ${ }^{5}$

A problem commonly occurring with the copolymerization method is the nonuniform distribution of functional groups throughout the bulk of the sample. Though overall composition of the formed particles matches that of the starting mixture, composition of fractions formed during the course of polymerization is often variable. A nonuniform surface results. ${ }^{5}$

Functional groups may be placed on the surface of microspheres after polymerization of the structural monomer. If the structural monomer is styrene, bromination and chloromethylation are suitable for the introduction of a wide range of functionalities. A major problem is the possibility of side reactions taking place on the polymer during aromatic substitution. Since the side products cannot be removed and their nature may not be known, effects on protein behavior are unpredictable. ${ }^{6}$ 
An alternate, straightforward method for the preparation of high-surface-area polystyrene and two copolymers of styrene is presented which has proved to be suitable for microcalorimetry. Adsorption of polymer from solution onto finely divided fumed silica produces characterizable, though nonspherical, substrates of high surface area. In addition, substrates with a range of surface properties sufficient to elicit different microcalorimetric responses during protein adsorption may be produced.

\section{MATERIALS AND METHODS}

\section{High-surface-area samples}

High-surface-area samples of polysytrene [MW 280,000 (GPC); Aldrich], poly (styrene-co-butyl methacrylate) [butyl methacrylate content 50\%, MW 200,000 (GPC); Scientific Polymer Products], and poly(styreneco-allyl alcohol) [alcohol content 5.4-6.0\%, MW 3000 (GPC); Scientific Polymer Products] were prepared by adsorbing polymer from solution onto finely divided fumed silica (Degussa) $\left(130 \mathrm{~m}^{2} / \mathrm{g}\right)$.

Preparation of silica

To remove surface water and standardize the surface with respect to the type of functional groups present, the silica was heated at $900^{\circ} \mathrm{C}$ for $10 \mathrm{~min}$ under vacuum. Heat-treated silica was kept sealed in a desiccator until used.

Preparation of high-surface-area polystyrene

Silica in the ratio $100 \mathrm{mg} / 100 \mathrm{ml}$ was added to a $2 \%$ solution of polystyrene in cyclohexane at $65^{\circ} \mathrm{C}$ and the mixture vigorously stirred for $20 \mathrm{~min}$. The sample was collected by passing the mixture through a coarse $(10-15 \mu \mathrm{m})$ fritted glass filter, and dried at $110^{\circ} \mathrm{C}$ under vacuum for $48 \mathrm{~h}$. The dried sample was ground with a mortar and pestle and stored in a capped glass bottle.

Preparation of high-surface-area poly(styrene-cobutyl methacrylate) and high-surface-area poly(styrene-co-allyl alcohol)

Copolymers with high surface areas were prepared in a way similar to that of polystyrene. Adsorption was allowed to occur from $1 \%$ solutions in toluene at $100^{\circ} \mathrm{C}$.

Verification of adsorption of polystyrene by silica

Verification was determined by noting a shift to lower wavenumbers of the surface silanol group after sample preparation. The shift indicates an increase in the effective mass associated with the silanol group and is qualitative evidence of physical bond formation. Heat-treated silica was added in the ratio $100 \mathrm{mg} /$ $100 \mathrm{ml}$ to each of a series of polystyrene/carbon tetrachloride solutions ranging in concentration from 0.001-3.0\%. After separation and drying, spectra of each sample were taken using Fourier-transform infrared spectroscopy.

\section{Adsorption isotherm}

The amount of adsorbed polymer or copolymer per gram of silica was determined from ratios of peak heights from infrared spectra. The heights of the polymer peak at $700 \mathrm{~cm}^{-1}$, attributed to out-of-plane deformation of the styrene aromatic group, 7 and the silica peak at $1100 \mathrm{~cm}^{-1}$, attributed to stretching of bulk Si$\mathrm{O}-\mathrm{Si}$ groups, ${ }^{8}$ were measured in absorbance relative to background at $1450 \mathrm{~cm}^{-1}$.

Calibration curves were made by taking spectra of mixtures of ground polymer or copolymer and silica in various proportions.

Transmission electron microscope study of prepared sample

To enhance electron density contrast of the carboncontaining polystyrene relative to the carbon film support grid, a staining agent was used. Ruthenium tetroxide is an oxidizing agent which apparently binds the phenyl group of polystyrene. ${ }^{9}$ A carbon grid dusted with a small amount of polystyrene-covered silica was vapor-stained for $1 \mathrm{~h}$ using a solution of $0.5 \% \mathrm{RuO}_{4}$ in deionized water.

Electron micrographs were taken with a JEOL 2000 electron microscope at an accelerating voltage of $400 \mathrm{kV}$.

Determination of minimum polystyrene thickness required to mask silicon oxide properties

Because of the composite nature of the high-surfacearea samples made for use in the microcalorimeter, it was necessary to ensure that any properties influencing the adsorption of protein were solely due to the polymer part of the sample without contribution from the silica. This was accomplished by determining the minimum polymer layer thickness at which contact angles became constant. Flat polymer samples were made beginning with undoped silicon wafers of crystallographic orientation [100] which were highly polished on both sides. An oxide layer of about $400 \mathrm{~A}$ was grown by heating the wafers at $1000^{\circ} \mathrm{C}$ in dry oxygen for $1 \mathrm{~h}$.

Wafers were cleaned by immersing for $20 \mathrm{~min}$ in a solution of one part ammonium hydroxide, one part $30 \%$ hydrogen peroxide, and five parts deionized wa- 
ter at $80^{\circ} \mathrm{C}$. After rinsing, the wafers were immersed for $20 \mathrm{~min}$ in one part hydrochloric acid, one part $30 \%$ hydrogen peroxide, and five parts deionized water at $80^{\circ} \mathrm{C}$, and rinsed again. To remove surface ions from the cleaning procedure, the resistivity of the rinse water was monitored until it matched that of uncontaminated deionized water.

Silicon oxide layer thicknesses of a series of $1 \times$ $1-\mathrm{cm}$ wafer pieces were measured using an ellipsometer and a one-layer software program for the silicon-silicon oxide system. This instrument and software allowed the measurement of extremely thin silicon oxide layers of assumed refractive index 1.464 to an accuracy of $\pm 3 \mathrm{~A}$.

Very thin polystyrene layers were placed on the oxide surfaces by adsorption from extremely dilute solutions in cyclohexane. From a second series of ellipsometer measurements an estimation of polymer layer thickness was made.

Half of the wafers were placed horizontally in a small glass container with flat sides and immersed in distilled octane. A small $(0.1-\mu \mathrm{l})$ drop of distilled, deionized water was placed at the center of each wafer. Contact angles were measured using a goniometer at 15 seconds after placement.

The remaining wafers were immersed in distilled, deionized water. Octane drops were placed on the underside of each wafer and the contact angles measured. Each series of contact angles was plotted against polystyrene layer thickness.

\section{Determination of surface area}

Surface areas of high-surface-area substrates were determined using a sorption analyzer. A nitrogenhelium $(0.293: 1)$ mixture at liquid nitrogen temperature was used as the adsorbate. Pretreatment included a 1-h outgassing with nitrogen at $150^{\circ} \mathrm{C}$.

\section{Interfacial energies}

\section{Contact angle measurements}

Contact angles of water on polystyrene and each copolymer were measured on flat samples prepared from $1 \%$ polymer solutions. A series of small $(0.1-\mu \mathrm{l})$ drops of distilled, deionized water were placed midway along each sample shortly after the drying step, and the angles measured.

\section{Pendant drop procedure}

Since stretching a solid surface does not result in a new surface with properties identical to the original, direct measurements of the surface tension of solids is not possible. The pendant drop method is suitable for measuring surface tensions of viscous liquids, and extrapolation of data to room temperature should give a reasonable estimate of the surface energy of the solid.

The experimental apparatus used for measuring surface tensions of molten polymer drops consisted of two vertical, 10 -inch-long aluminum tubes situated one inside the other. A glass syringe was placed so that a polymer drop could be formed midway along the length of the inner tube. Temperature at the drop position was maintained to within $\pm 0.2^{\circ} \mathrm{C}$ of the set point up to $170^{\circ} \mathrm{C}$ by a controller attached to band heaters situated above and below viewing holes. Temperature at the drop position was monitored with a thermocouple placed adjacent to the drop. To maintain a relatively oxygen-free environment, dry nitrogen was continually flushed through the inside tube.

The temperature range over which each material was tested was chosen to provide reasonable times for drop equilibration. Polystyrene (MW 280,000) was quite viscous and was tested at higher temperatures than was poly(styrene-co-allyl alcohol) (MW 3000), which was fluid just a few degrees above $T_{g}$. At the starting temperature for each material, the drop was allowed to stand for $18 \mathrm{~h}$. At relatively high temperatures where the material was fluid, and at all temperatures for poly(styrene-co-allyl alcohol), 5-min equilibration times were found to be sufficient.

At each temperature, the drop was allowed to sit until its shape did not change. Shape parameters were determined from measurements of photographic negatives projected over a large distance. Surface tensions were calculated from the equation:

$$
\gamma=\frac{g \rho\left(d_{e}\right)^{2}}{H}
$$

where $g$ is the gravitational acceleration, $\rho$ the density of the drop, $d_{e}$ the equatorial diameter of the drop, and $1 / H$ a correction factor determined by the shape of the drop. ${ }^{10}$ Andreas ${ }^{11}$ tabulated $1 / H$ as a function of the shape parameter $S$ defined as the ratio $d_{s} / d_{e}$, where $d_{s}$ is the horizontal diameter of the drop measured at a distance $d_{e}$ up from the bottom of the drop. Surface tensions at $20^{\circ} \mathrm{C}$ were estimated by extrapolation.

\section{Determination of polymer densities}

The density of a nonporous solid can be found by using a pycnometer of known volume and a noninteracting liquid of known density. For use in calculating surface tensions, densities of each polymer were measured over the same temperature intervals as were pendant drops. The liquid chosen for this study was polydimethylsiloxane (1000 centistokes), which is stable in the temperature range of $70-170^{\circ} \mathrm{C}$. It also did not appear to interact with the polymers studied over the length of time required to complete measurements. 
The volume of a $10-\mathrm{ml}$ pycnometer as a function of temperature was determined in the temperature range $70-170^{\circ} \mathrm{C}$ using polydimethylsiloxane along with density vs. temperature data supplied by the manufacturer.

Temperature was followed by monitoring voltage using a digital multimeter and a copper-constantan thermocouple, one end of which was placed inside the pycnometer, and the other in an ice-water reference.

Densities of polymers were determined over the temperature range $70-170^{\circ} \mathrm{C}$ by placing $5 \mathrm{~g}$ of polymer or copolymer in the pycnometer along with polydimethylsiloxane. The following relation was used to determine density at each temperature:

$$
\begin{aligned}
& (\text { Density })_{\text {Total }} \\
& =(\text { Volume fraction })_{\text {Liquid }} \cdot(\text { Density })_{\text {Liquid }} \\
& \quad+(\text { Volume Fraction })_{\text {Polymer }} \cdot(\text { Density })_{\text {Polymer. }} .
\end{aligned}
$$

Densities at $20^{\circ} \mathrm{C}$ were obtained by extrapolation.

\section{Wilhelmy plate}

A Wilhelmy plate study was carried out to verify that polymer surfaces have time-dependent properties when placed in contact with water. This study required the measurement of very small forces exerted on flat plates by water during vertical plate movement. These measurements were accomplished by fitting an Instron Universal Testing machine with a 10-g load cell and by use of an auxiliary plotter connected to the circuitry of the Instron's chart recorder. This setup allowed a reversible, variable-speed vertical drive for plate movement, and a one-way sweeping variable speed recorder with a sufficiently small full-scale range of about $3 \mathrm{~g}$. The system was calibrated using metal wires of known masses.

We coated $1 \times 1$-inch cleaned oxide-covered silicon wafers with either a thick (order of micrometers) or thin (order of nanometers) layer of polymer or copolymer by allowing adsorption from solution, followed by drying at $110^{\circ} \mathrm{C}$ for $18 \mathrm{~h}$ under vacuum. A wafer was attached by wire to the load cell and lowered at a rate of $0.5 \mathrm{~cm} / \mathrm{min}$ into a beaker of distilled, deionized water to a distance of about $0.8 \mathrm{~cm}$; the direction of the Instron was reversed and the plate pulled upward from the water at the same rate. This procedure resulted in a recorded hysteresis loop. The plate was then immersed in distilled, deionized water and removed at varying intervals to be tested on the Instron. Water used for soaking was changed after each test. Testing continued for up to $30 \mathrm{~h}$ of immersion time.

\section{Microcalorimetry}

A preliminary study was carried out to determine the suitability of the prepared high-surface-area sub- strates for microcalorimetry. Purified hen egg-white lysozyme (Sigma Chemical Co., St. Louis, MO) (3× crystallized $)^{12}$ in $0.1 \mathrm{M}$ phosphate buffer, $\mathrm{pH} 7.14$, was allowed to adsorb onto prewetted substrates of total surface area $4.65 \mathrm{~m}^{2}$. Heats of adsorption were obtained using a modified Tien-Calvet microcalorimeter ${ }^{13}$ at $37^{\circ} \mathrm{C}$ for a range of protein solution concentrations. The amount of lysozyme adsorbed during reaction in the calorimeter was calculated from absorbance measurements of the supernatant protein solution at $280 \mathrm{~nm}$ and a calibration curve. A detailed description of these procedures is provided elsewhere. ${ }^{14}$

\section{RESULTS}

\section{Sample preparation}

The infrared spectrum of nonporous fumed silica in the as-received state showed a broad absorption band centered around $3400 \mathrm{~cm}^{-1}$ attributable to adsorbed water vapor, and a lower-intensity, asymmetrical peak at $3750 \mathrm{~cm}^{-1}$ attributable to the fundamental stretching frequency of a hydroxyl group attached to a silicon atom on the surface of the silica ${ }^{15}$ [Fig. 1(a)]. The width and asymmetry of this peak indicated a distribution of configurations of the silanol group.

Heat treatment under vacuum resulted in large part in the disappearance of the water peak and an increase in symmetry of the silanol peak at $3750 \mathrm{~cm}^{-1}$ [Fig. 1(b)]. These changes indicate that adsorbed water associates directly with silanol groups, and that its removal leaves the silanol groups with essentially identical environments.

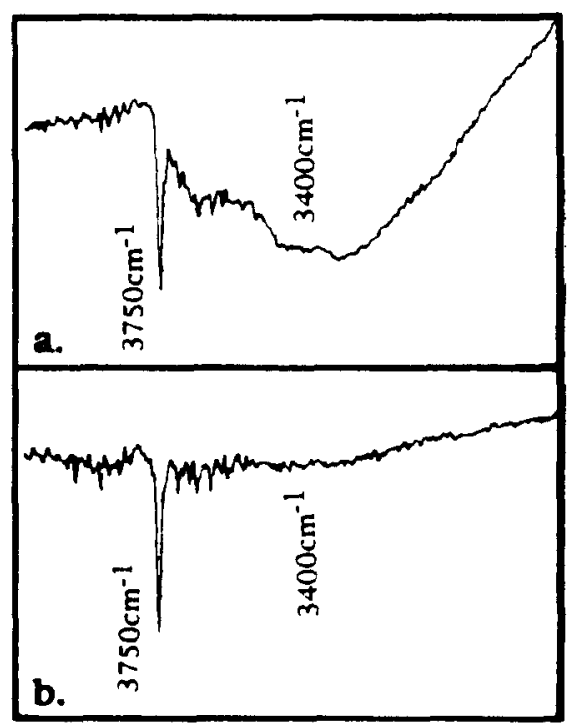

Figure 1. Infrared spectra. (a) Fumed silica, as received. (b) Fumed silica, heat-treated at $900^{\circ} \mathrm{C}$ for $10 \mathrm{~min}$ in vacuum. 


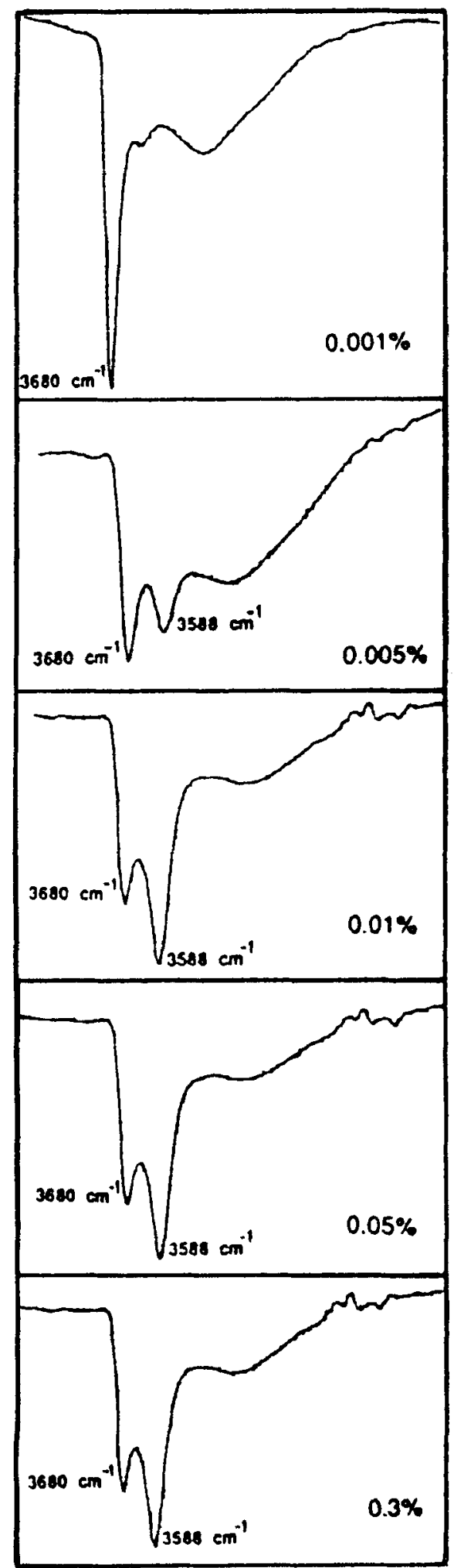

Figure 2. Infrared spectra showing progress of adsorption. of polystyrene by silica at concentrations of $0.001-3.0 \%$ in carbon tetrachloride. The shift of the silanol peak indicates physical bond formation with polystyrene.
The series of spectra in Figure 2 shows the progress of adsorption of polystyrene by silica. Little adsorption occurred from very dilute polystyrene solutions such as $0.001 \%$. The appearance of the small peak at $3588 \mathrm{~cm}^{-1}$ indicates that a few surface silanol groups were physically associated with polystyrene. Relative peak heights changed as solution concentration increased, indicating a larger fraction of bonded surface groups. No apparent change in ratio was noted at concentrations above $0.01 \%$, indicating that some silanol groups probably remained unbonded for steric reasons. The constant ratio also suggests that given the uniformity of the silanol groups in the starting condition, uniform coverage of the silica surface by polystyrene was achieved.

The polystyrene adsorption isotherm was of the high-affinity type, with a limiting plateau of $0.70 \mathrm{~g} / \mathrm{g}$ silica starting at a low bulk concentration of $0.02 \%$ (Fig. 3). Isotherms of copolymers did not show limiting plateaus. Cohen-Stuart et al. ${ }^{16}$ attributed isotherms with continuously increasing adsorbed amounts to broad molecular weight distributions. Polydispersity indexes for poly(styrene-co-butyl methacrylate) and poly(styrene-co-allyl alcohol) were 1.88 and 2.33 , re-
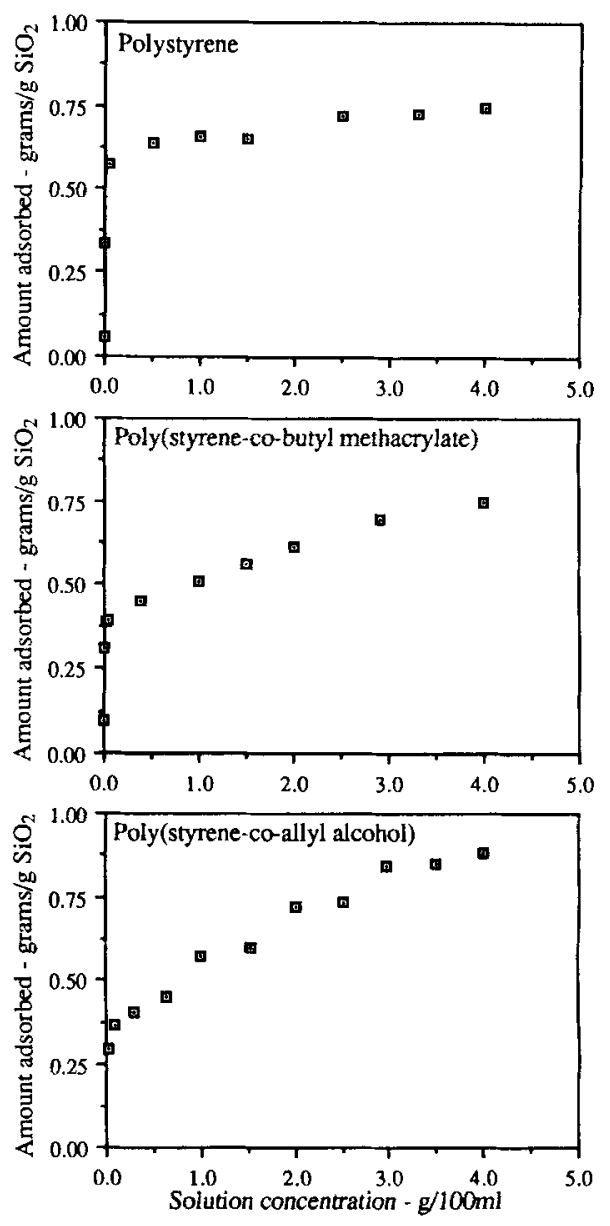

Figure 3. Adsorption isotherms of sample polymers on silica. 


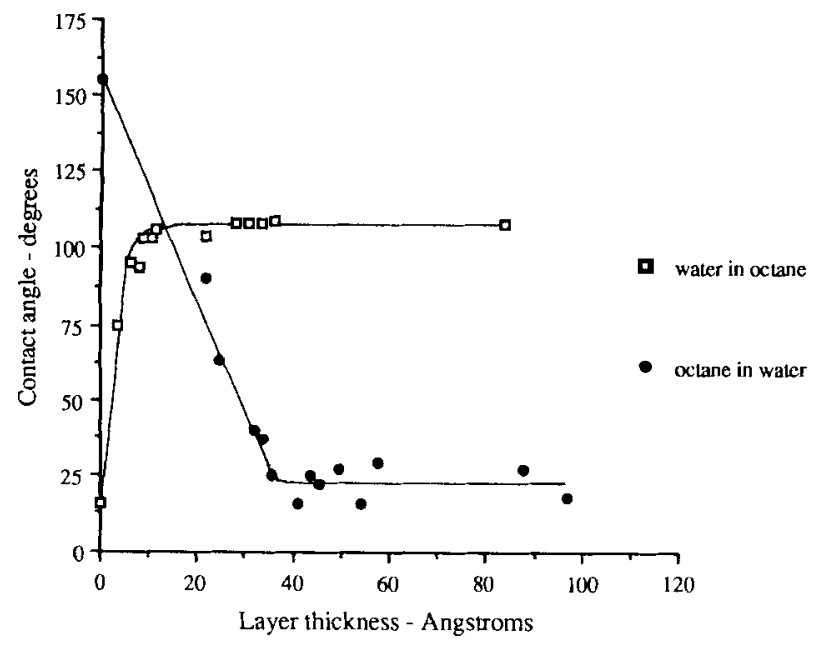

Figure 4. Contact angles as a function of thickness of polystyrene layer.

spectively. ${ }^{17}$ The index for polystyrene was not available.

Surface areas of high-surface-area polystyrene, poly(styrene-co-butyl methacrylate), and poly(styrene-coallyl alcohol) were determined to be 71,93 , and $85 \mathrm{~m}^{2}$ / $\mathrm{g}$, respectively.

Contact angles remained constant beyond a minimum polymer layer thickness (Fig. 4). The leveling-off of water contact angles at smaller apparent thicknesses can possibly be attributed to swelling of the polystyrene layer upon immersion in octane. It appears that a polystyrene layer thickness of $35 \mathrm{~A}$ was sufficient to provide a surface uninfluenced by silicon oxide properties.

From the electron micrograph (Fig. 5), polystyrenecoated silica appears as clumps approximately $50 \mathrm{~nm}$ in diameter, fused into clusters. The smallest cluster found on the grid measured $350 \mathrm{~nm}$.

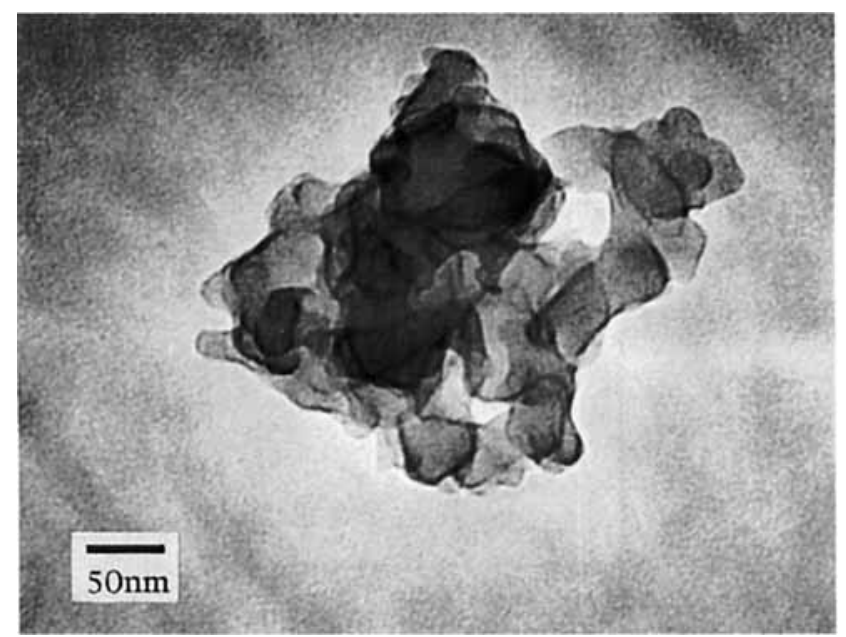

Figure 5. Transmission electron micrograph: polystyrenecoated silica, (original magnification $\times 150,000$ ).
Calculation from adsorption isotherm data predicted layer thicknesses for polystyrene, poly(styrene$c o$-butyl methacrylate), and poly(styrene- $c 0$-allyl alcohol) of 50, 37, and $41 \mathrm{~A}$, assuming measured densities of $1.08,1.07$, and $1.09 \mathrm{~g} / \mathrm{cm}^{3}$, respectively (Fig. 6), and even coverage of silica.

\section{Interfacial energies}

Water and methylene iodide contact angles and estimates of the polar and dispersion components of the sample polymers using these data and Wu's harmonic mean approximation ${ }^{18}$ are reported in Table I. Higher water contact angles on the surface of polystyrene and lower angles in the case of poly(styrene-co-allyl alcohol) indicate more complete wetting of the poly(styrene-co-allyl alcohol) surface by water.

Polar and dispersion components and the polarity for polystyrene show good agreement with those found by $\mathrm{Wu}$ using the same method. The highest polarity, $\gamma^{p} / \gamma$, was predicted for poly(styrene-cobutyle methacrylate). Comparable data could not be found for the copolymers.

An alternate method for obtaining the polar and dispersion components of the sample polymers for use in the harmonic mean relationship follows. Contact angles on the solid were made using a liquid whose main interactions are dispersion. Methylene iodide has a very low polar component of $1.3 \mathrm{ergs} / \mathrm{cm}^{218}$ and a high surface tension of $58.0 \mathrm{ergs} / \mathrm{cm}^{2}$. The dispersion component of the solid can thus be directly estimated using a geometric mean approximation. The polar component is then the difference from surface energies determined from pendant drops (Table II). Using method 2, the largest polarity was predicted for poly(styrene-co-allyl alcohol).

Surface tensions of sample polymers as measured by the pendant drop method are shown in Figure 7. The

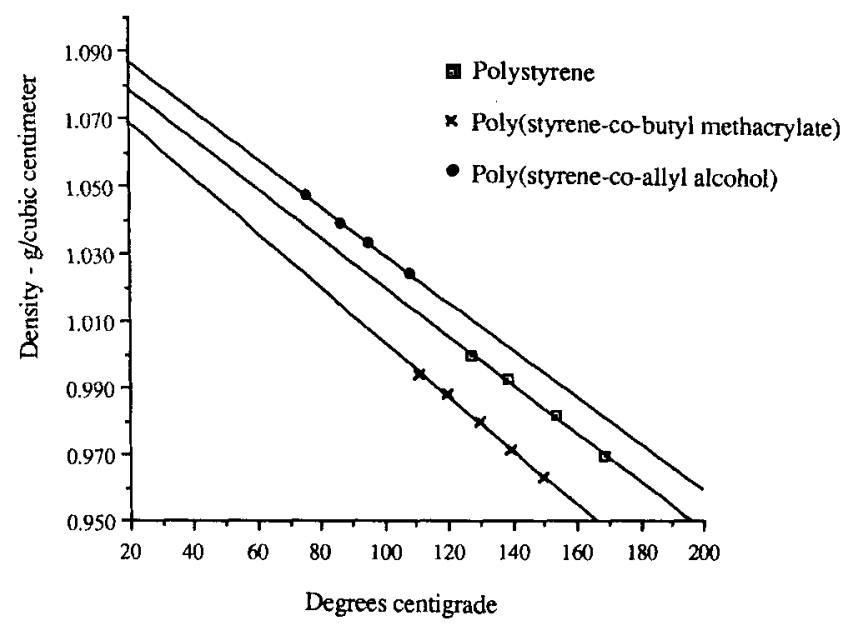

Figure 6. Polymer densities as a function of temperature. 
TABLE I

Method 1: Surface and Component Energies for Sample Polymers from Contact Angles and Harmonic Mean Approximation

\begin{tabular}{|c|c|c|c|c|c|c|}
\hline \multirow[b]{2}{*}{ Surface } & \multicolumn{2}{|c|}{ Contact Angle (degrees) } & \multicolumn{3}{|c|}{ Ergs $/ \mathrm{cm}^{2}$} & \multirow[b]{2}{*}{$\gamma^{p} / \gamma$} \\
\hline & Water & Methylene Iodide & $\gamma^{d}$ & $\gamma^{p}$ & $\gamma$ & \\
\hline Polystyrene & $90.3 \pm 0.8$ & $38.3 \pm 1.8$ & 38.2 & 4.6 & 42.8 & 0.11 \\
\hline Poly(styrene-co-butyl methacrylate) & $86.5 \pm 1.9$ & $55.3 \pm 2.9$ & 30.4 & 7.6 & 38.0 & 0.20 \\
\hline Poly(styrene-co-allyl alcohol) & $80.5 \pm 1.5$ & $29.8 \pm 1.5$ & 41.5 & 8.9 & 50.4 & 0.18 \\
\hline
\end{tabular}

data showed good linearity. The extrapolated value for polystyrene at $20^{\circ} \mathrm{C}, 46.0 \mathrm{ergs} / \mathrm{cm}^{2}$, was high compared to that found by $\mathrm{Wu}\left(40.7 \mathrm{ergs} / \mathrm{cm}^{2}\right)$ using a similar method. ${ }^{19}$ The density found in this work, $1.08 \mathrm{~g} / \mathrm{cm}^{3}$ at $20^{\circ} \mathrm{C}$, agrees closely with that used by $\mathrm{Wu}$, at $1.06 \mathrm{~g} / \mathrm{cm}^{3}$.

Surface tensions from pendant drops for poly(styrene-co-butyl methacrylate) and poly(styrene-coallyl alcohol) were found in this work to be 37.2 and $55.1 \mathrm{ergs} / \mathrm{cm}^{2}$, respectively.

Interfacial energies using the two methods are shown in Table III. Both methods predicted polystyrene to have the highest interfacial energy, and method 2 predicted poly(styrene-co-allyl alcohol) to have the lowest.

\section{Wilhelmy plate}

Figure 8(a) shows a curve obtained when a cleaned, oxide-covered silicon wafer with no polymer layer was tested using the Wilhelmy plate method. No hysteresis was seen. This result demonstrates the adequacy of the wafer cleaning procedure and indicates that the wafers, highly polished on both sides, have no roughness detectable by the test method.

A typical hysteresis loop obtained from a polymercovered wafer is shown in Figure 8(b). The initial increase in force recorded as the plate touched the water was a result of the mass of water present in the meniscus due to wetting. As the plate was immersed further, the force decreased from buoyancy effects. The change in slope represents the point where the advancing contact angle reached a maximum, after which it remained constant. As the plate was removed from the water, the force increased from decreasing buoyancy effects.
The change in slope represents the point where the contact angle, which is the receding contact angle, reached a minimum. Forces were obtained through extrapolation of the lines representing constant contact angles to zero depth of immersion. Advancing and receding contact angles were calculated using the following relation ${ }^{20}$ :

$$
F=m g+P \gamma_{L} \cos \theta
$$

where $F$ is the measured force, $m g$ the force due to gravity, $P$ the perimeter of the plate, $\gamma_{L}$ the surface tension of water, and $\theta$ the contact angle. The force required for calculation is actually a difference of forces on the plate at the points of zero depth of immersion and before immersion. The $m g$ term therefore cancels out.

Significant hysteresis and continued change in both advancing and receding contact angles were noted for all samples for the 30-h duration of the test (Fig. 9).

The difference between advancing and receding angles at $30 \mathrm{~h}$ ranged from $40-50^{\circ}$. Smith found hysteresis using the Wilhelmy plate method to range from $35-50^{\circ}$ for polybutadiene films of controlled surface characteristics. ${ }^{21}$ Good and Kotsidas found a hysteresis of $26^{\circ}$ using a sessile bubble technique with crosslinked polystyrene. ${ }^{22}$

As testing time approached $30 \mathrm{~h}$, changes in the curves slowed. A water contact time of $48 \mathrm{~h}$ was assumed to be sufficient for sample equilibration.

\section{Microcalorimetry}

From the adsorption isotherms and adsorption heats for the three substrates [Fig. 10(a-c)], two trends are apparent. As bulk lysozyme concentration increased,

TABLE II

Method 2: Surface and Component Energies for Sample Polymers from Contact Angles, Geometric Mean Approximation, and Pendant Drop Results

\begin{tabular}{lccccr}
\hline \multicolumn{1}{c}{ Surface } & Contact Angle (degrees): & \multicolumn{3}{c}{ Ergs $/ \mathrm{cm}^{2}$} \\
\cline { 3 - 5 } & Methylene lodide & $\gamma^{d}$ & $\gamma^{p}$ & $\gamma$ & $\gamma^{p} / \gamma$ \\
\hline Polystyrene & $38.3 \pm 1.8$ & 41.0 & 5.0 & 46.0 & 0.11 \\
Poly(styrene-co-butyl methacrylate) & $55.3 \pm 2.9$ & 32.1 & 5.1 & 37.2 & 0.14 \\
Poly(styrene-co-allyl alcohol) & $29.8 \pm 1.5$ & 45.5 & 9.6 & 55.1 & 0.17 \\
\hline
\end{tabular}




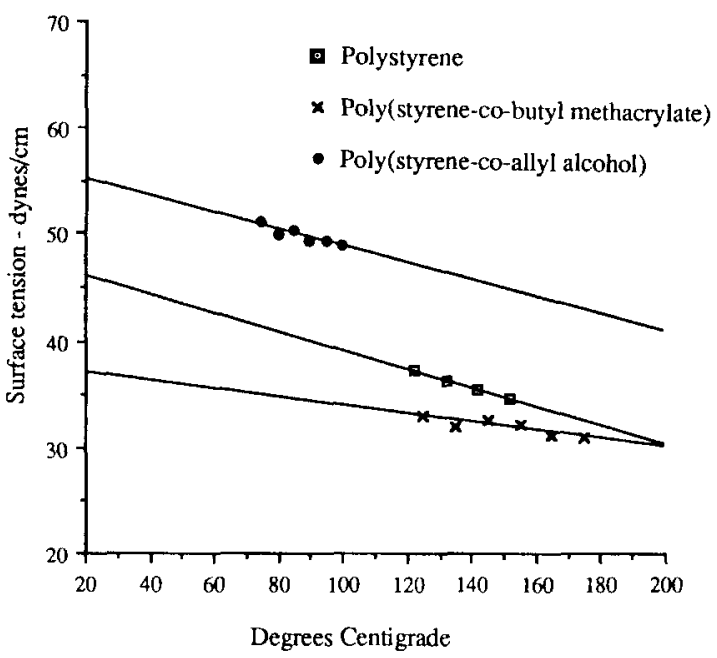

Figure 7. Surface tensions of polymers as a function of temperature determined by pendant drop method. $95 \%$ confidence intervals for $y$ at $x=20^{\circ} \mathrm{C}$ : polystyrene $(42.7$, 49.2), poly(styrene-co-butyl methacrylate) (33.2, 41.0), poly(styrene-co-allyl alcohol) $(51.9,58.2)$.

the amount of lysozyme adsorbed by substrate followed a step pattern with a series of plateaus showing successively higher adsorbed amounts. In addition, in the cases of polystyrene and poly(styrene-co-butyl methacrylate), adsorption heats showed endothermic discontinuities at each rise between plateaus. Heats at other points, including those at zero protein concentration, were exothermic and apparently due to dilution of buffer ions by pure water in the sample holder. With poly(styrene-co-allyl alcohol), no discontinuities were found, and all heats were of the magnitude of dilution heats.

No discernible differences were noted in the amounts of lysozyme adsorbed by the three substrates.

\section{DISCUSSION}

Fowkes $^{23}$ proposed that surface energy may be considered to be a sum of terms, each due to a single type of intermolecular force, i.e., dipolar, dispersion,

TABLE III

Interfacial Energies

\begin{tabular}{lcc}
\hline \multicolumn{1}{c}{ Surface } & Method $1^{*}$ & Method 2 \\
\hline Polystyrene & 46.4 & 43.7 \\
Poly(styrene-co-butyl methacrylate) & 32.5 & 39.5 \\
Poly(styrene-co-allyl alcohol) & 40.3 & 36.6 \\
\hline
\end{tabular}

${ }^{*} \gamma^{d}$ and $\gamma^{p}$ determined from harmonic mean equation.

${ }^{t} \gamma^{d}$ and $\gamma^{p}$ determined from geometric mean equation and pendant drop results. Data are ergs $/ \mathrm{cm}^{2}$.

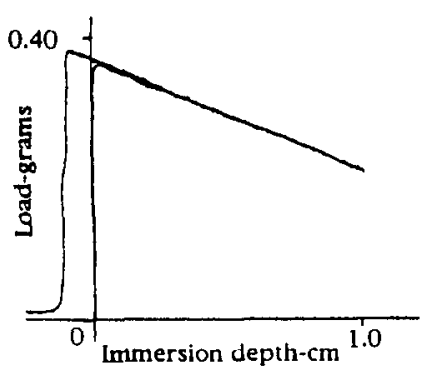

(a)

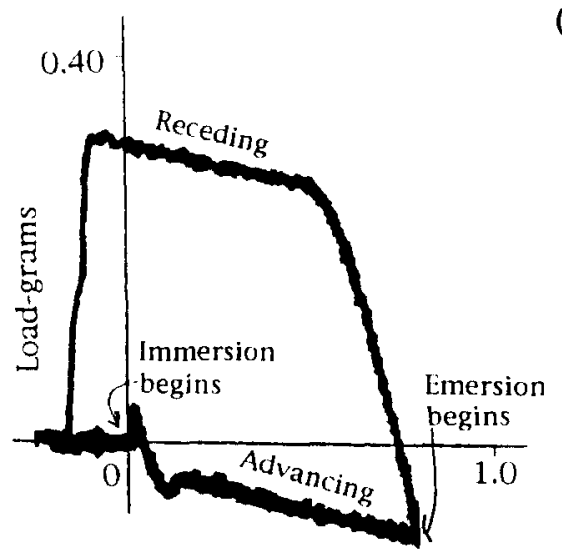

(b)

Immersion depth-cm

Figure 8. (a) Wilhelmy hysteresis loop obtained from cleaned, oxide-covered silicon wafer. No hysteresis is seen. (b) Wilhelmy hysteresis loop.

metallic, etc. Where two phases meet, forces of a similar kind operate across the interface, reducing the interfacial energy. The net interfacial energy is then the result of surface forces which are not able to operate across the interface. It has been proposed that interfacial energy may be of importance in predicting the behavior of proteins upon adsorption at an interface. ${ }^{24}$

Higher water contact angles on the surface of polystyrene and lower angles on the surface of polystyreneco-allyl alcohol) suggest that polar forces operate across the interface to a larger extent in the poly(styrene-coallyl alcohol)-water system than occurs with the other two materials.

Both geometric and harmonic means produce widely accepted estimates of the dispersion component in low-energy systems such as polymers in water. Both means can be derived from London's theory. Using method 1, simultaneous solution of two harmonic mean equations in combination with Young's equation, $\gamma_{l g} \cos \theta=\gamma_{s g}-\gamma_{s l}$ yielded both polar and dispersion components. The ranking of dispersion components obtained for the three polymers using this method was identical to that obtained using method 2, where dispersion components were determined directly from a geometric mean and the contact angles of methylene iodide. Both means produced rankings 
(a)

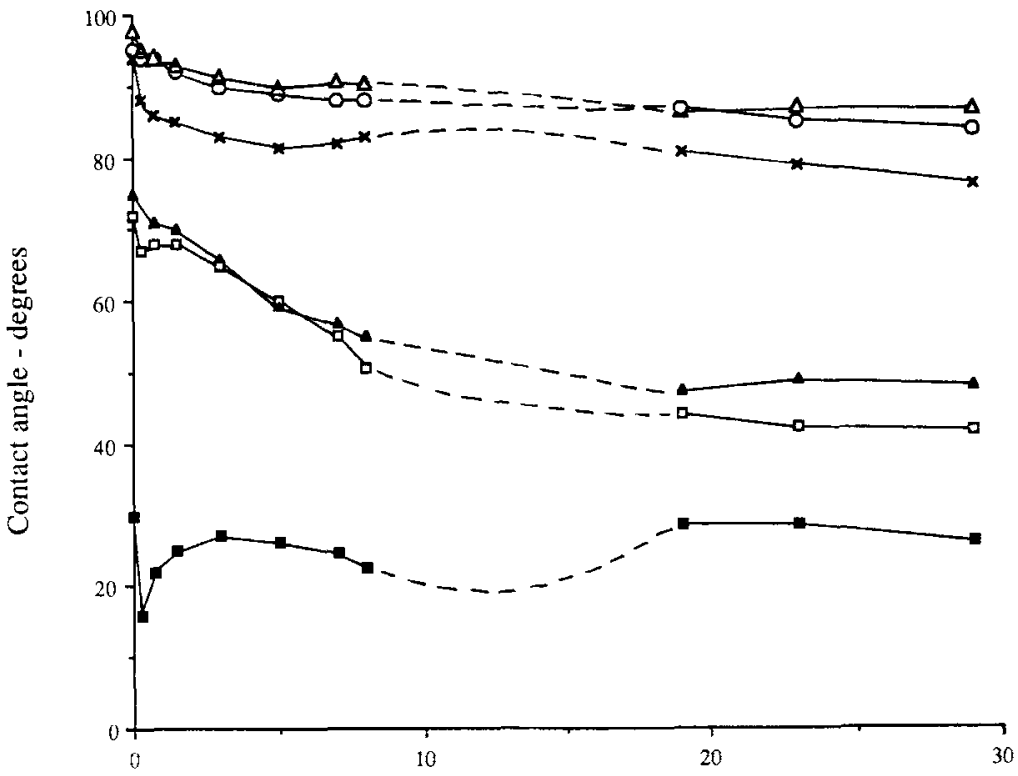

(b)

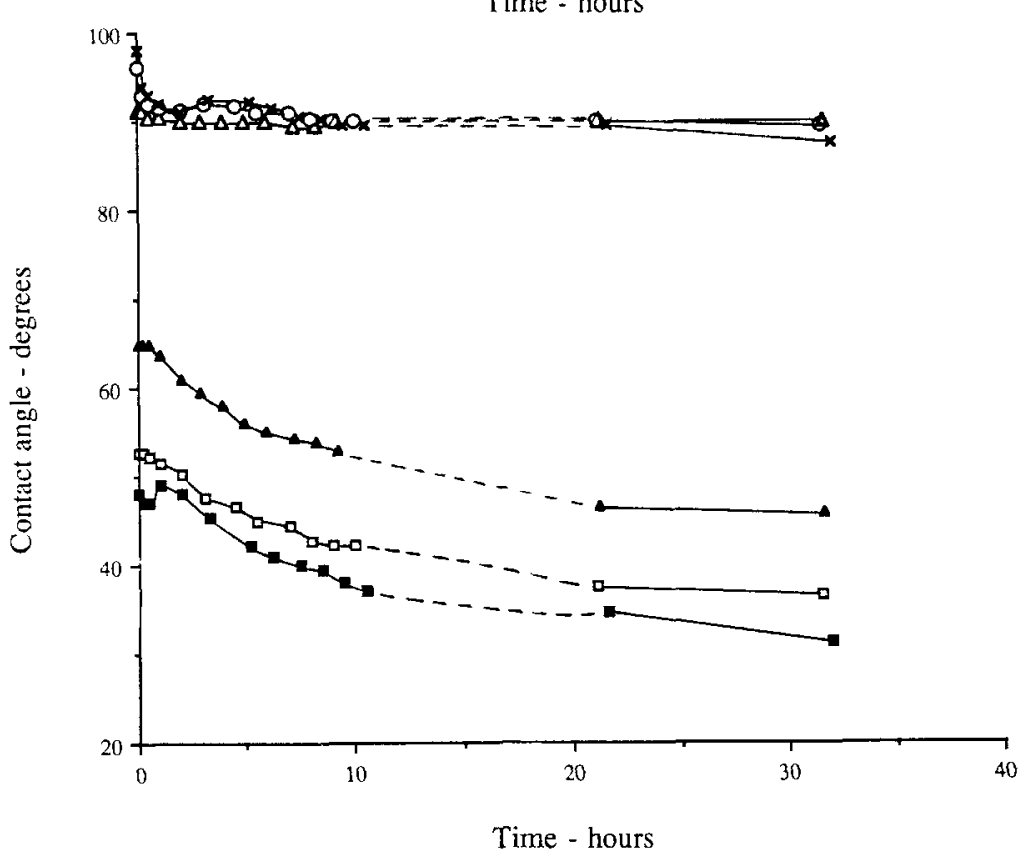

Figure 9. Contact angles vs. immersion time in water from Wilhelmy plate results. (Top) Thick samples. (Bottom) Thin samples. Advancing angles: $\triangle$, polystyrene; $O$, poly(styrene-co-butyl methacrylate); $X$, poly(styrene-co-allyl alcohol). Receding angles: $\Delta$, polystyrene; $\square$, poly(styrene-co-butyl methacrylate); $\mathbf{\omega}$, poly(styrene-co-allyl alcohol). Dotted lines indicate time intervals over which no measurements were taken.

identical to those of the polymer densities, which is expected.

In contrast, at present no acceptable expression for polar interactions is available. While theoretically it can be shown that under very specific conditions the polar component can be described by a geometric mean, in practice the geometric mean approximation when used with contact angles routinely over- or underestimates the contribution from the polar component. From situations where direct measurements can be made, $\mathrm{Wu}$ determined that a harmonic mean yields a more accurate estimate of the polar component in low-energy systems than does the geometric mean. The harmonic mean estimation of polar components in method 1 allowed the highest polarity, $\gamma^{p} / \gamma$, to be predicted for poly(styrene-co-butyl methacrylate), which does not correlate with water contact angles on the three materials.

A more direct estimate of the polar components, method 2, taken to be the difference between surface 
(a)

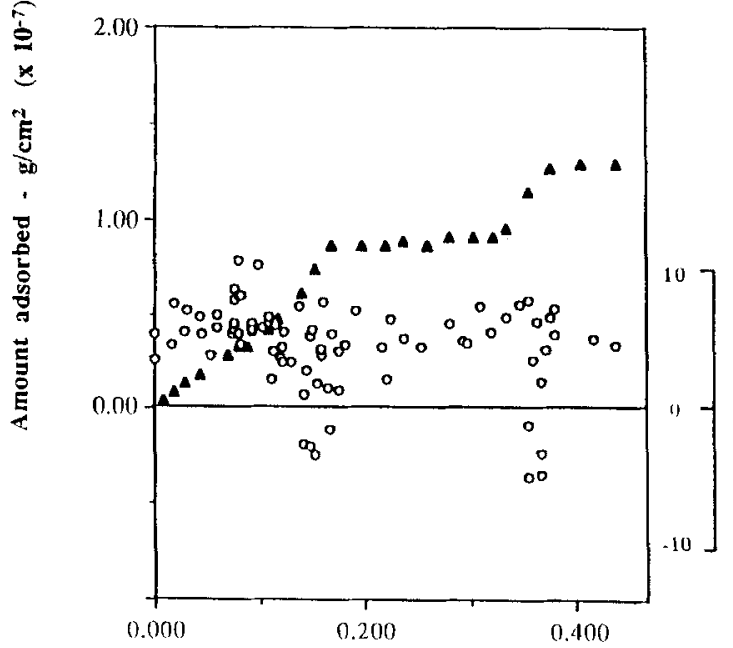

Protein concentration - g/100ml

(b)

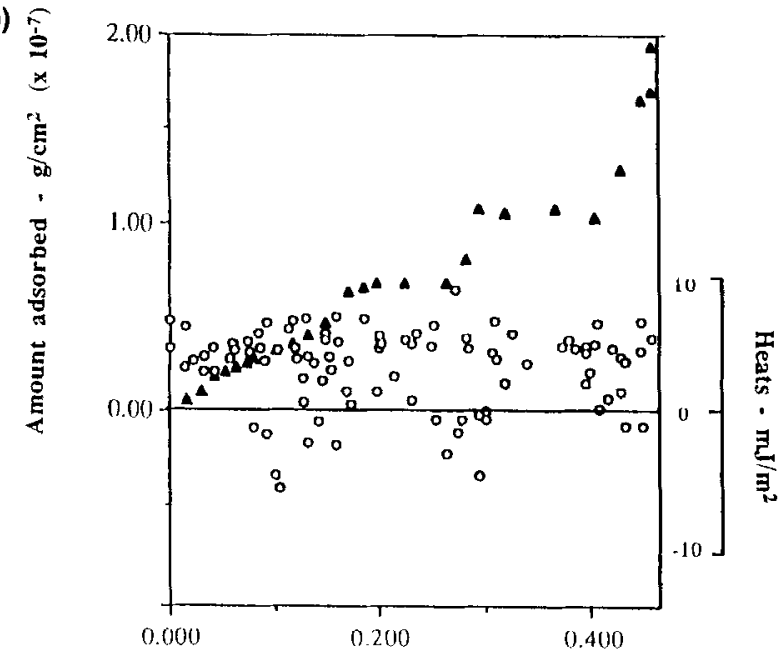

(c)

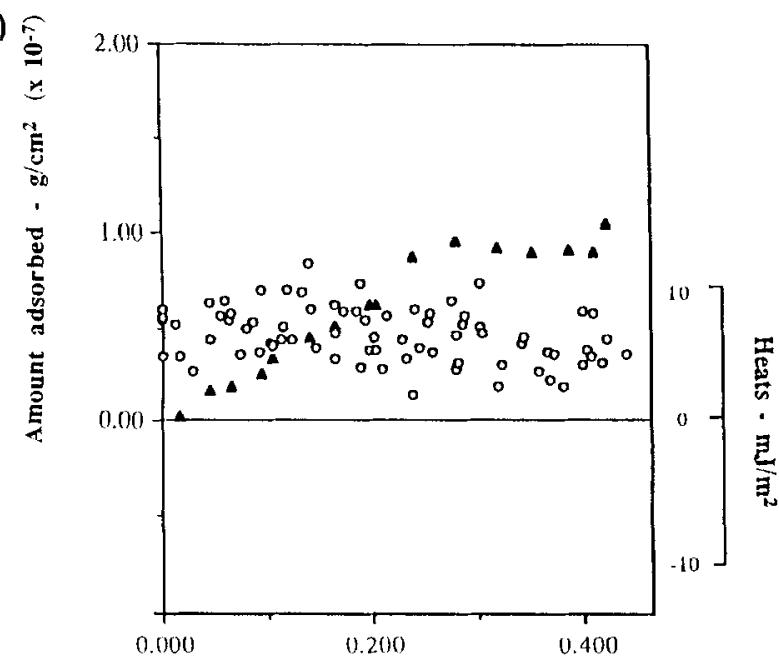

Protein concentration - g/100mi

Figure 10. Adsorption isotherms $(\boldsymbol{\Delta})$ and microcalorimetric heats $(O)$ for (a) polystyrene, (b) poly(styrene-co-butyl methacrylate), and (c) poly(styrene-co-allyl alcohol) at ionic strength $0.1 \mathrm{M}$ from phosphate buffer. energies measured from pendant drops and a geometric mean estimation of the dispersion component, predicted that the highest polarity occurs with poly(styrene-co-allyl alcohol), which agrees well with water contact angles on the three materials.

Polar and dispersion components determined by the above two methods were used in Wu's harmonic mean relationship to estimate polymer-water interfacial energies:

$$
\gamma_{12}=\gamma_{1}+\gamma_{2}-\frac{4 \gamma_{1}^{d} \gamma_{2}^{d}}{\gamma_{1}^{d}+\gamma_{2}^{d}}-\frac{4 \gamma_{1}^{p} \gamma_{2}^{p}}{\gamma_{1}^{p}+\gamma_{2}^{p}}
$$

where $\gamma_{12}$ is the interfacial energy, $\gamma_{1}$ the polymer surface energy as determined from pendant drops, and $\gamma_{2}$ the surface tension of water. The $\gamma_{1,2}^{p, d}$ terms are the polar and dispersion components of the polymer and of water. The value of the surface tension of water is from Lide. ${ }^{25}$ The polar and dispersion components of water are those used by Fowkes, who estimated the dispersion component from measurements of the interfacial tension of water against a series of nonpolar liquids of known surface tensions, and applied a geometric mean approximation. ${ }^{23}$ The surface tension and components of methylene iodide are those used by $\mathrm{Wu}$, who estimated the component tensions from known surface and interfacial tensions of water and methylene iodide, and the harmonic mean approximation. ${ }^{18}$

Both methods ranked the polystyrene-water system to have the highest interfacial energy, and method 2 ranked poly(styrene-co-allyl alcohol)-water to have the lowest. In either case, the three materials provided only a narrow range of interfacial properties.

The above interfacial energies were determined for air-equilibrated surfaces. Biomedical polymers, however, while generally processed in normal atmospheres, encounter an aqueous environment during use. A Wilhelmy plate study verifies the change in interfacial properties as a function of contact time with water.

An ideal surface has a single equilibrium contact angle. Contact angle hysteresis occurs when the surface is nonideal. Most surfaces are complicated and are not uniform, plane or nondeformable. Two fairly reproducible contact angles in such cases are the largest, or the advancing angle, and the smallest, or the receding angle. Langmuir ${ }^{26}$ proposed that hysteresis is the result of the overturning of molecules during contact with the wetting liquid. Andrade ${ }^{27}$ suggested that hysteresis results from penetration of the liquid during its advancement over the surface, leaving a more wettable surface during the receding angle test. In effect, surfaces which have not reached equilibrium with the wetting liquid are expected to show contact angle hysteresis.

Polymers, as a consequence of weak intermolecular bonds, are able to reorient groups near the surface 
when placed in contact with a new environment. In the relatively hydrophobic air environment, polar groups may be buried in the bulk of the polymer, while in water the same polar groups may be oriented toward the water phase. The driving force for reorientation is the minimization of energy between the two phases.

The first specimens tested were dry. During the course of the test, sample-water contact time was about $2 \mathrm{~min}$. Subsequent tests resulted in a decrease in both advancing and receding contact angles, suggesting a more wettable surface. Water penetration and plasticization of the region near the surface may have promoted motions of chain segments and side groups, resulting in reorientation of polar groups or segments toward the water phase. Despite the hydrophobic nature of the material, water penetration of the surface is not unlikely, because of the low molecular volume of water.

Changes seen in both advancing and receding curves reflect changes in surface wettability. Large fluctuations seen in the receding curves may reflect the presence of molecular motions in the polymer layer. If this is so, the fluctuations are consistent with the sensitivity of receding angles to small changes in surface wettability as noted by Johnson and Dettre. ${ }^{20}$ From studies of a series of two-phase materials of varying surface composition, Johnson and Dettre found that advancing angles remained high despite a surface composition of over $95 \%$ wettable phase. Receding angles, however, decreased considerably with the introduction into the surface of just $5 \%$ wettable phase.

The systematic trends noted in the protein adsorption isotherms of the three substrates and the pattern of adsorption heats of polystyrene and poly(styreneco-butyl methacrylate) indicate that the substrates as prepared are of sufficient surface area to be useful in microcalorimetry. The substantial difference in the patterns of heats noted in the cases of polystyrene and poly(styrene-co-butyl methacrylate) as compared to poly(styrene-co-allyl alcohol) indicates that samples prepared by the method described can have surface characteristics of sufficient variety that differences in the adsorption heats of one protein may be discerned. Complete data and possible interpretations of the observed trends will be presented in a subsequent paper.

\section{SUMMARY}

The preparation and characterization of highsurface-area polymeric substrates suitable for the microcalorimetry of protein adsorption are described. High-surface-area polystyrene, poly(styrene-co-butyl methacrylate), and poly(styrene-co-allyl alcohol) were prepared by adsorbing polymer from solution onto finely divided fumed silica. Verification of adsorption of polymer by silica was determined by noting peak shifts of the surface silanol group in the infrared. The minimum thickness of polystyrene required to mask silicon oxide properties was found to be about $35 \mathrm{~A}$. Substrate surfaces were characterized by estimating the polymer-water interfacial energies from pendant drops and two contact angle methods. The suitability of the substrates for microcalorimetry was demonstrated by adsorption studies of lysozyme using a modified Tien-Calvet microcalorimeter and noting trends in the resulting heats.

\section{References}

1. R. B. Merrifield, "Solid phase peptide synthesis. I. The synthesis of a tetrapeptide," J. Am. Chem. Soc., 85, 21492154 (1963).

2. A. Rembaum and Z. A. Toke, eds., Microspheres: Medical and Biological Applications, CRC Press, Boca Raton, 1988, pp. 65-67.

3. S. M. Moghimi, L. Illum, and S. S. Davis, "Physiological and physicochemical considerations in targeting of colloids and drug carriers to the bone marrow," Crit. Rev. Ther. Drug Carrier Syst., 7, 187-209 (1990).

4. R. Madison, J. D. Maklis, and C. Thies, "Latex nanosphere delivery system," Brain Res., 552, 90-98 (1990).

5. R. Arshady, "Microspheres for biomedical applications: Preparation of reactive and labelled microspheres," Biomaterials, 14, 5-15 (1993).

6. R. Arshady, "Beaded polymer supports and gels. II. Physico-chemical criteria and functionalization," J. Chromatogr., 586, 198-219 (1991).

7. Diana L. Hanson, ed., Sprouse Collection of Infrared Spectra, Book 1: Polymers, Sprouse Scientific Systems, Paoli, PA, 1987, p. 162.

8. Clara D. Craver, ed., Desk Book of Infrared Spectra, Coblentz Society, Norwalk, CT, 1986, p. 423.

9. J. S. Trent, J. I. Scheinbeim, and P. R. Couchman, "Ruthenium tetroxide staining of polymers for electron microscopy," Macromolecules, 16, 589-598 (1983).

10. A. W. Adamson, Physical Chemistry of Surfaces, 4th ed., Wiley, New York, 1982, p. 28.

11. J. M. Andreas, E. A. Hauser, and W. B. Tucker, "Boundary tension by pendant drops," J. Phys. Chem., 42, 1001 (1938)

12. V.P. Saxena and D. B. Wetlaufer, "Formation of threedimensional structure in proteins. I. Rapid nonenzyme reactivation of reduced lysozyme," Biochemistry, 9, 5015-5022 (1970).

13. S. H. Maron and F. E. Filisko, "A modified Tien-Calvet microcalorimeter for polymer solution measurements," J. Macromol. Sci. Phys., B6, 57-77 (1972).

14. V. A. Lee, "Microcalorimetry of the adsorption of lysozyme onto uncharged substrates," Ph.D. Dissertation, University of Michigan, 1993.

15. R.S. McDonald, "Surface functionality of amorphous silica by infrared spectroscopy," J. Phys. Chem., 62, 11681178 (1958)

16. M. A. Cohen-Stuart, H. M. Scheutjens, and G. J. Fleer, "Polydispersity effects and the interpretation of polymer adsorption isotherms," J. Polymer Sci. Polymer Phys., 18, 559 (1980). 
17. Scientific Polymer Products, personal communication, January 4, 1993.

18. S. Wu, "Polar and nonpolar interactions in adhesion," J. Adhesion, 5, 39-55 (1973).

19. S. Wu. "Surface and interfacial tensions of polymer melts," J. Phys. Chem., 74, 632-638 (1970).

20. R. W. Johnson and R. H. Dettre, "Wettability and contact angles," in Surface and Colloid Science, Vol. 2, E. Matijevic (ed.), Wiley, New York, 1969, pp. 85-153.

21. L. Smith, C. Doyle, D. E. Gregonis, and J. D. Andrade, "Surface oxidation of cis-trans polybutadiene," J. Appl. Polymer Sci., 27, 1269-1276 (1982).

22. R. J. Good and E. D. Kotsidas, "The contact angle of water on polystyrene: A study of the cause of hysteresis," J. Colloid Interface Sci., 66, 360-362 (1978).
23. F. M. Fowkes, "Attractive forces at interfaces," Industrial and Engineering Chemistry, 56, 40-52 (1964).

24. E. Ruckenstein and S. V. Gourisankar, "Preparation and characterization of thin film surface coatings for biological environments," Biomaterials, 7, 403-422 (1986).

25. D. R. Lide, ed., CRC Handbook of Chemistry and Physics, 71st ed., Boca Raton, 1990-1991, pp. 6-8.

26. I. Langmuir, "Overturning and anchoring of monolayers," Science, 87, 493-500 (1938).

27. J. D. Andrade, L. M. Smith, and D. E. Gregonis, Surface and Interfacial Aspects of Biomedical Polymers, Vol. 1, Plenum, New York, 1985, pp. 262-265.

Received December 12, 1994

Accepted September 6, 1995 